\title{
Comprehensible Explanations of Determination Methods of Three Basic System Properties and Examples
}

\author{
Wenhong Liu \\ School of Electronic Information, Shanghai Dianji University, Shanghai, China
}

Email address:

420375509@qq.com

To cite this article:

Wenhong Liu. Comprehensible Explanations of Determination Methods of Three Basic System Properties and Examples. Higher Education Research. Vol. 9, No. 4, 2021, pp. 93-97. doi: 10.11648/j.her.20210604.13

Received: August 16, 2021; Accepted: September 4, 2021; Published: September 10, 2021

\begin{abstract}
In the course of learning Signals and Systems, it is one of the emphases and difficulties to determine the basic system properties, such as linearity, time-invariance and causality. In teaching, it is found that students often only know the written expression of the determination methods, but do not understand the core of the determination methods. So they feel difficult to use. In order to help students to understand, this paper focuses on giving popular and common explanations to the traditional determination methods of the above three basic system properties, rather than sticking to the rigor of the theory. As for linearity determination, from the embodiment of additivity and homogeneity, the input signals are constructed; the discrimination of time invariance is considered from two aspects: time delay and the transformation of signals by systems; it is more convenience for judgment of causality to obtain the time relationship of output and input from some special moments. By means of the comprehensible explanations of the determination methods in this paper, students can easily understand and master the traditional determination methods of three basic system properties. More importantly, they can apply it to solve the determination problems of linearity, time invariance and causality of systems.
\end{abstract}

Keywords: System Properties, Linearity, Time-Invariance, Causality, Determination Methods Interpretations

\section{Introduction}

Signals and Systems is a very important basic course for electronic information and related professional fields, such as communication engineering, etc [1]. On the one hand, it plays a bridge role between public basic courses and specialized courses [2]; On the other hand, it is also the very necessary foundation of many related professional courses, such as Digital signal processing, communication principle, automatic control principle and so on. In addition, with the development of science and technology and the integration of disciplines, the concepts, basic theories and analytical methods involved in the course of Signals and Systems not only gradually become one of the most basic concepts and methods in many fields of science and engineering, but also play an increasingly important role in almost every field of science and technology [3-9]. Even in the economic finance and humanities and social sciences also have a wide range of applications [1, 10].

For students majoring in electronic information engineering and communication engineering, Signals and Systems is one of the most basic courses. In many key universities of electronic information majors of the postgraduate entrance examination, Signals and Systems will be listed as the entrance examination of professional compulsory courses. Students pay more attention to this course, have the desire to learn, but often have a feeling of awe. On the one hand, their the prerequisite courses such as advanced mathematics, engineering mathematics, circuit analysis and other prerequisite courses are not solid; On the other hand, there are many theoretical formulas involved in this course, which are not much easy to understand. Even if you understand some of them, you still can't really use them. For example, judging the fundamental nature of systems is one of the key and difficult points of this course [6-8]. For the properties of a system such as linearity, time-invariance and causality, judgments are given in the textbook, the definition of the literal can know, but a brief examples and explain process are not given [9]. For linearity, the general formula given is too abstract, and the two basic elements of linearity, superposition and homogeneity, are not emphasized enough [9]. For time invariance, it is not very clear what the system does, what is time varying and what is constant. With regard to causality, students will be 
confused about how to consider some special moments to determine whether the system is non-causality without learning more general methods of discrimination. It are not easy to understand these, students don't understand why will have this or that determine the steps, so often is rote learning these properties of some specific systems, rather than application.

This paper does not stick to the rigorous theoretical analysis, from the perspective of helping students understand, to explore new teaching methods. Add own analysis and understanding by referring to many versions of textbooks and videos [1, 11-14]. Firstly, based on the basic concept of signal, the main function of the system and the internal connection between the signal and the system, the common determination methods of linearity, time-invariance and causality of the system are mainly explained. Then, combined with many examples of students often feel difficult to judge, respectively explain; Finally, based on many typical examples, detailed descriptions have shown for the causality of linear time-invariance to further improve the students' ability to grasp this part of the content [15].

\section{Three Basic Properties of a System}

In the course of learning Signals and Systems, it is one of the emphases and difficulties to determine the basic system properties, such as linearity, time-invariance and causality. A system model is followed in Figure 1.

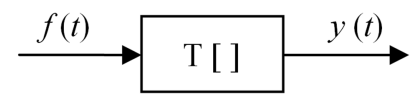

Figure 1. Block diagram representation of a system.

Let us discuss three basic properties of a system. Basically, focus on their definitions and determinations.

\subsection{Linearity Definition and Determination}

A linear system is one that has both homogeneity (or uniformity) and superposition. The homogeneity of the system means that when the input excitation changes by a factor of a, the response of the output also changes by a factor of a, where $\mathrm{a}$ is an arbitrary constant. The superposition of a system means that the total response of the system is equal to the sum of the response components generated by each excitation acting on the system [3].

Linearity Definition: the system possesses the important property of superposition (the weighted sum).

Additivity property means that the response to $f_{1}(t)+f_{2}(t)$ is $y_{1}(t)+y_{2}(t)$.

Scaling or homogeneity property means that the response to $a f_{1}(t)$ is $a y_{1}(t)$, where $a$ is any complex constant, $a \neq 0$.

How to determine the linearity based on the definition? Considering the two properties defining a linear system, input signal can be constructed as following

$$
f(t)=a f_{1}(t)+b f_{2}(t)
$$

Then, determining whether or not output is

$$
y(t)=a y_{1}(t)+b y_{2}(t)
$$

Yes, the system is linear.

\subsection{Time-invariance Definition and Determination}

If the system is in zero state, the relationship between the output response and the input excitation does not change with the time of the input excitation on the system, it is a constant system [8].

Time-invariance definition: Characteristics of the system are fixed over time. Time-invariant system is that if $f(t) \rightarrow y(t)$, then $f\left(t-t_{0}\right) \rightarrow y\left(t-t_{0}\right)$.

Next, we give the steps to determine the time-invariance of a system.

There is a system $y(t)=\mathrm{T}[f(t)]$.

Step 1: focus on time.

Let input time $t$ delayed to $t-t_{0}$, the output corresponding to this time input is

$$
y_{1}\left(t-t_{0}\right)=\mathrm{T}\left[f\left(t-t_{0}\right)\right]
$$

Step 2: focus on the function of the system.

Consider the input $f(t)$ obtained by shift $f(t)$ in time: $f(t)=f\left(t-t_{0}\right)$, the input is

$$
y_{2}\left(t-t_{0}\right)=\mathrm{T}\left[f\left(t-t_{0}\right)\right]
$$

If

$$
y_{1}\left(t-t_{0}\right)=y_{2}\left(t-t_{0}\right)
$$

the system is time invariant.

\subsection{Causality Definition and Determination}

For any input signal, a system has causality if its output signal value at any time depends only on the input signal value at that time and at previous times, and is independent of the input signal value at future times [5].

Definition of the Causality: a system is causal if the output at any time depends only on values of the input at the present time and in the past.

When a system is suspected of being non-causal, an approach to showing this that is often very useful is to seek a counterexample to use our intuition to find an special time on which the condition of causality is violated.

Special time may be $t=0,-2,-1,1,2 \ldots$.

\section{Explanations and Examples}

In teaching, it is found that students often only know the written expression of the determination methods, but do not understand the core of the determination methods. So they feel it is difficult to use.

In order to help students to understand, this paper focuses 
on giving popular and common explanations to the traditional determination methods of the above three basic system properties, rather than sticking to the rigor of the theory.

As for linearity determination, from the embodiment of additivity and homogeneity, the input signals are constructed;

The discrimination of time invariance is considered from two aspects: time delay and the transformation of signals by systems;

It is more convenience for judgment of causality to obtain the time relationship of output and input from some special moments.

By means of the comprehensible explanations of the determination methods in this paper, students can easily understand and master the traditional determination methods of three basic system properties.

More importantly, they can apply them to solve the determination problems of linearity, time invariance and causality of systems.

The explanations of the determination methods of three basic system properties, linearity, time-invariance and causality, in this paper, are comprehensible and it is easy to understand and operate for students.

\subsection{Linear or Not}

Example 1: Considering a system $S$ whose input $f(t)$ and output $y(t)$ are related by

$$
y(t)=t^{2} f(t)
$$

To determine whether or not $\mathrm{S}$ is linear.

We consider two arbitrary inputs $f_{1}(t)$ and $f_{2}(t)$.

$$
\begin{aligned}
& f_{1}(t) \rightarrow y_{1}(t)=t^{2} f_{1}(t) \\
& f_{2}(t) \rightarrow y_{2}(t)=t^{2} f_{2}(t)
\end{aligned}
$$

Constructing input $f(t)$ is a linear combination of $f_{1}(t)$ and $f_{2}(t)$. That is

$$
f(t)=a f_{1}(t)+b f_{2}(t)
$$

where $a$ and $b$ are arbitrary scalars. Then the corresponding output $y(t)$ can be expressed as

$$
\begin{aligned}
y(t) & =t^{2} f(t) \\
& =t^{2}\left[a f_{1}(t)+b f_{2}(t)\right] \\
& =a t^{2} f_{1}(t)+b t^{2} f_{2}(t) \\
& =a y_{1}(t)+b y_{2}(t)
\end{aligned}
$$

We conclude that the system $S$ is linear.

Example 2: Let us apply the linearity-checking procedure of the previous example to another system $S$ whose input $f(t)$ and output $y(t)$ are related by

$$
y(t)=f^{2}(t)
$$

To determine whether or not $S$ is linear.

Considering two arbitrary inputs $f_{1}(t)$ and $f_{2}(t)$.

$$
\begin{aligned}
& f_{1}(t) \rightarrow y_{1}(t)=f_{1}^{2}(t) \\
& f_{2}(t) \rightarrow y_{2}(t)=f_{2}^{2}(t)
\end{aligned}
$$

Let input $f(t)=a f_{1}(t)+b f_{2}(t)$, and output

$$
\begin{aligned}
y(t) & =f^{2}(t) \\
& =\left[a f_{1}(t)+b f_{2}(t)\right]^{2} \\
& =a^{2} f_{1}^{2}(t)+2 a b f_{1}(t) f_{2}(t)+b^{2} f_{2}^{2}(t) \\
& =a^{2} y_{1}(t)+2 a b f_{1}(t) f_{2}(t)+b^{2} y_{2}(t) \\
& \neq a y_{1}(t)+b y_{2}(t)
\end{aligned}
$$

Clearly, when input $f(t)=a f_{1}(t)+b f_{2}(t)$, the $y(t)$ is not equal to $a y_{1}(t)+b y_{2}(t)$.

So, we conclude that the system $S$ is not linear.

\subsection{Time Invariant or Not}

If a system has time-varying parameters, it must be not time invariance. So, we focus on constant coefficients systems.

Example 3: $y(t)=f(t-1)$

Let input time $t$ delayed to $t-t_{0}$, the output corresponding to this time input is

$$
y_{1}\left(t-t_{0}\right)=f\left(t-t_{0}-1\right)
$$

Consider the input $f(t)=f\left(t-t_{0}\right)$, obtained by shift, the output corresponding to this input is

$$
y_{2}\left(t-t_{0}\right)=f\left(t-t_{0}-1\right)
$$

We see that

$$
y_{2}\left(t-t_{0}\right)=y_{1}\left(t-t_{0}\right)
$$

So this system is time-invariant.

Example 4: $y(t)=f(1-t)$

Let input time $t$ delayed to $t-t_{0}$, the output corresponding to this time input is

$$
y_{1}\left(t-t_{0}\right)=f\left[1-\left(t-t_{0}\right)\right]=f\left(1-t+t_{0}\right)
$$

Consider the input $f(t)=f\left(t-t_{0}\right)$, obtained by shift, the output corresponding to this input is

$$
y_{2}\left(t-t_{0}\right)=f\left(1-t-t_{0}\right)
$$

We see that

$$
y_{2}\left(t-t_{0}\right) \neq y_{1}\left(t-t_{0}\right)
$$

So this system is time-varying. 
Example 5: $y(t)=f(2 t)$ is following as figure 2 .

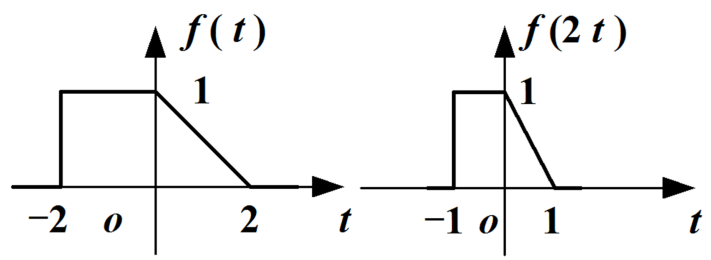

Figure 2. Example 5: $y(t)=f(2 t)$.

Step 1: focus on time. $t$ delayed to $t-t_{0}$

$$
y_{1}\left(t-t_{0}\right)=f\left[2\left(t-t_{0}\right)\right]=f\left(2 t-2 t_{0}\right)
$$

Step 2: focus on the function of system. $f(t)=f\left(t-t_{0}\right)$

$$
y_{2}\left(t-t_{0}\right)=f\left(2 t-t_{0}\right)
$$

Because $y_{2}\left(t-t_{0}\right) \neq y_{1}\left(t-t_{0}\right)$, the system is not time invariant.

\subsection{Causal or Not}

Examples of causal system:

$$
\begin{array}{r}
y(t)=f(t-1) \\
y(t)=g(t) f(t) \\
y(t)=f(t) \cos (t+1)
\end{array}
$$

The systems are causal because the output at any time depends only on values of the input at the present time and in the past.

Examples of non-causal system:

$$
y(t)=f(t+1)
$$

Because $y(0)=f(1)$, that means the output at $t=0$ depends on the future input at $t=1$.

Try by yourself:

$$
\begin{aligned}
& y(t)=f(-t), \quad t=-1 \\
& y(-1)=f(1) \\
& y(t)=f(2 t), \quad t=1 \\
& y(1)=f(2) \\
& y(t)=f\left(\frac{t}{2}\right), \quad t=-2 \\
& y(-2)=f(-1)
\end{aligned}
$$

These systems are not causal.

\section{Conclusions}

For the judgment of linearity, emphasis is placed on the consideration of homogeneity and superposition to construct the input signal. For the judgment of time invariance, emphasis on finding the input signal, respectively from the system to the signal transformation or processing effect, and time delay on the input signal, output signal of the same effect, two perspectives to understand the system time invariance; The judgment of causality, for a system with constant coefficients, determines the temporal relationship between the output signal and the input signal through some critical moment points, such as, etc., or finds a counterexample to determine its non-causality. Finally, taking the second-order linear differential equation with constant coefficients as an example, the detailed common determination process of its linearity, time-invariance and causality is given, so as to deepen the understanding of the basic determination method of the important properties of common systems.

In this paper, from the point of view that help students understand not tight in theory, the basic characteristics of the three commonly used methods, the key to the interpretation of the popular, makes up for the many teaching materials in the interpretation of this part is too simple, general, such as "obviously", instead of the decision process, the students don't know why the results. This paper explains the determination methods, so that students can easily understand and master the three basic properties of the common determination methods, to use them to determine the linear, time-invariance and causality of common systems learning objectives. Related teaching for the three basic properties of the system, giving the analysis and judgment process of thought and steps, has been carried out for several years of teaching practice. According to the students' learning effect, this popular use of explanation is easy to understand and master for students [15].

\section{Acknowledgements}

This work was partly supported by the Shanghai Dianji University Research Projects (No. 20B074, 19B283, 17 B56 and 16B141), and Shanghai Dianji University Key English Course Project (Signals and Systems) and Shanghai Municipal Government Key Online-to-Offline Hybrid Course Project (Signals and Systems).

\section{References}

[1] Alan V. Oppenheim, Alan S. Willsky, and S. Hamid Nawab, Signals and Systems, 2nd ed., Publishing House of Electronics Industry, 2012, pp. 44-57.

[2] Simon Haykin and Barry Van Veen, Signals and Systems, 2nd ed., Publishing House of Electronics Industry, 2012, pp. 5569.

[3] Guan zhizhong, Xia Gongke, and Meng Qiao, Signals and Linear Systems, 6th ed., Higher Education Press, 2015.

[4] Zheng Junli, Ying Qiheng, and Yang Weili, Signals and Linear Systems, 3rd ed., Higher Education Press, 2011.

[5] Xu Shoushi, Tan Yong, and Guo Wu, Signals and Systems Theory Method and Application, 3rd ed., University of Science and Technology of China Press, 2018. 
[6] Guo Baolong, Yan Yunyi, Zhu Juanjuan, and Wu Xianxiang, Engineering Signals and Systems, Higher Education Press, 2014.

[7] Chen Shentan, Guo Baolong, Li Xuewu, and Gao Jianning, Signals and Systems, 4th ed., Xidian University Press, 2018.

[8] Chen Houjin, Hu Jian, Xue Jian, and Li Jvpeng, Signals and Systems, Higher Education Press, 2020.

[9] Zhang Xiaohong, Signals and Systems, 4th ed., Xidian University Press, 2018.

[10] Liu Shufan et al., Signal and System Operation, Simulation and Comprehensive Design Experiment, Machinery Industry Press, 2010 .
[11] https://mooc1-1.chaoxing.com/course/207603919.html.

[12] Guo Baolong, http://www.icourses.cn/sCourse/course_4233.html.

[13] Meng Qiao http://www.icourses.cn/sCourse/course_2596.html

[14] Chen http://www.icourses.cn/sCourse/course_3343.html.

Houjin,

[15] Liu Wenhong, https://mooc1.chaoxing.com/mycourse/teachercourse?moocId $=215717567 \&$ clazzid $=34814981 \&$ edit $=$ true $\& v=0 \&$ cpi $=0 \& p a$ geHeader $=0$. 